\title{
Leptospirosis Nephropathy
}

National Cancer Institute

\section{Source}

National Cancer Institute. Leptospirosis Nephropathy. NCI Thesaurus. Code C123029.

Interstitial nephritis due to Leptospira, which may be associated with non-oliguric acute kidney injury. 